ten zwischen Nichtwählern und Wählern hinsichtlich soziostruktureller Eigenschaften (S. 86 - 141) und politischer Einstellungen (S. 143 - 199) herausgearbeitet. Der Erkenntnisgewinn zur Frage, ob Nichtwähler eine Gefahr für die Demokratie darstellen, fällt demgegenüber weniger ergiebig aus. Der um Ausgewogenheit bemühten Würdigung des Nichtwählens als „Krisensymptom“ (S. 204) oder als Gefährdung der „demokratische(n) Qualität“, jedoch nicht als Gefahr für die „,institutionelle Ordnung“ (S. 206), kommt einige Plausibilität zu. Sie bleibt aber gleichzeitig vage und sowohl empirisch als auch argumentativ nicht durchgängig nachvollziehbar. Mit der Empfehlung, „bewährte und neue gesellschaftliche und politische Inklusionsstrategien zu nutzen, um die Kongruenz zwischen Beteiligten und Betroffenen (wieder-)herzustellen“ (S. 207), schließt de Nève ihre Studie ab. Wie diese Strategien, vor dem Hintergrund eines gestiegenen Niveaus an politischer Unübersichtlichkeit in politischen Mehrebenensystemen (institutionelle und inhaltliche Interdependenzen, Verhandlungscharakter politischer Entscheidungsprozesse, damit einhergehend Intransparenz und unklarere politische Verantwortungszuschreibung) aussehen und implementiert werden können, wird eine wichtige Aufgabe weiterer Forschungsarbeiten sein.

Benjamin Höhne

\title{
Neugefasstes Standardwerk zur Parteienfinanzierung
}

\section{Sickinger, Hubert: Politikfinanzierung in Österreich. Ein Handbuch, Czernin Verlag, Wien} 2009, 527 Seiten, € 39,-.

Für die komparatistische Analyse eines international selten bearbeiteten Themenfeldes (und die Finanzen politischer Parteien sind ein in der Parteienforschung durchweg vernachlässigter Aspekt) haben sich vier aufeinander folgende Schritte als nützlich erwiesen. Zunächst bereiten mehr oder weniger kompatible Aufsatzsammlungen das Feld vor, dann folgen ausgefeilte Studien einzelner Länder, später vergleichende Arbeiten über Ländergruppen. Schließlich wird eine Zusammenfassung und Systematisierung der so gewonnenen Einsichten möglich. Als das hier angezeigte Werk erstmals erschien, war sein Verfasser bereits durch das Buch „Politisches Geld“ (1990, mit Rainer Nick) als profunder Kenner der Materie ausgewiesen. Das neue Buch von Hubert Sickinger war und ist nicht nur umfangreich, sondern auch umfassend. Das gilt ganz besonders für die breite, im besten Sinne erschöpfende, aber nicht ermüdende Abhandlung aller Teilaspekte des Themas. Als Oberbegriff für die monetär quantifizierbaren Kosten der Parteiendemokratie umfasst Politikfinanzierung neben den (facettenreich dargebotenen) Parteifinanzen auch Politikergehälter und die Kosten der parlamentarischen Infrastruktur.

Mit seinem Handbuch stellte sich Sickinger schon 1997 in eine Reihe mit den Klassikern des Genre: Alexander Heard (USA, 1960), Khayyam Z. Paltiel (Kanada, 1970), Uwe Schleth (Deutschland, 1973) und Michael Pinto-Duschinsky (Großbritannien, 1981) hatten die Stufe der länderspezifischen Intensivstudien bereits überzeugend eingeleitet. Später ergänzten dann Stefan Hofmann (Spanien, 1998), Marcin Walecki (Polen, 2005) und Martin Schurig (Frankreich, 2006) die Reihe der für einen fundierten Vergleich von Parteifinanzen unverzichtbaren Länderstudien. Jetzt legt Sickinger eine grundlegend überarbeitete und vollständig aktualisierte Neufassung seines Standardwerks über Österreich vor. 
Die (dritte) Stufe des Vergleichs von zwei bis vier Ländern wurde auf breiter Front erst in diesem Jahrhundert erreicht, als in größerer Zahl entsprechende Studien erschienen: Doris Cordes (2001), Michael Brändle (2002), Ingrid van Biezen (2003), Claire Smith (2005), Kevin Casas-Zamora (2005) und Michael Koß (2008). Gudrun Klee-Kruse (1993) und Andrea Römmele (1995) waren allerdings auch heute noch beachtenswerte Vorläufer gewesen. Jetzt ist auch die vierte Stufe - konzeptionell angelegt anspruchsvolle Vergleiche einer größeren Zahl von Demokratien - möglich geworden. Es wird aber wohl noch dauern, bis solche Analysen die durch Sickinger vorgeführte Breite und Tiefe der Durchdringung des Gegenstandes erreichen.

Dem Anspruch eines Handbuches entspricht die weitgehend additive Gliederung. Über zwanzig Kapitel behandeln in unterschiedlicher Länge und sachlich bedingter Ausdifferenzierung am österreichischen Beispiel, aber durchaus komparatistisch, die verschiedenen Geldquellen für politische Aktivität, Finanzvolumen, Vergleichsmaßstäbe, Entwicklungstrends und Reformperspektiven. In insgesamt 18 Kapiteln präsentiert der Autor alle denkbaren Facetten der Politikfinanzierung in einer kleinen, aber als Beispiel zugleich besonders wichtigen Demokratie. Der deutsche „Parteienstaat" und die italienische „partitocrazia“ sind dort eine herausragende Verbindung eingegangen; nirgendwo ist die Mediatisierung von gesellschaftlichen Organisationen und politischen Institutionen durch die Parteien so ausgeprägt wie in Österreich. Anders als in Deutschland haben deshalb die seit Jahren rückläufigen Mitgliederzahlen nicht zu einem vergleichbaren Rückbau der Parteiorganisation geführt. Sickinger erörtert die Graswurzelfinanzierung der Politik durch Mitgliedsbeiträge und Kleinspenden ebenso wie die plutokratische Finanzierung durch Spenden von Einzelunternehmen und Verbänden, Parteiunternehmen und Kreditaufnahme, Politikerbezüge und „Parteisteuern“ (in Deutschland verschämt Mandatsträgerabgaben genannt), die einzelnen Arten der direkten staatlichen Parteienfinanzierung in Bund, Ländern und Gemeinden sowie auf EU-Ebene und in den Parlamenten, indirekte Formen der Parteienfinanzierung aus öffentlichen Kassen, Volumen, Trends und Auswirkungen der staatlichen Finanzierung, die Einnahmenprofile der einzelnen Parteien, Wahlkampfkosten und einen detaillierten Anforderungskatalog für eine Reform der landesüblichen Politikfinanzierung.

In vier Kapiteln werden relevante Grundlageninformationen zum Thema ausgebreitet: die Einordnung in die Parteienforschung, die Beziehungen zur Korruption, das landesspezifische Parteiensystem, der Staatsaufbau und das System der Interessenvermittlung sowie die staatsrechtlichen Rahmenbedingungen. Eine knappe Einleitung und ein noch kürzeres Nachwort runden die Darstellung ab. Das Literaturverzeichnis erschließt auf insgesamt 25 Druckseiten die gesamte internationale Literatur zum Thema, auch wenn, dem spezifischen Gegenstand (Länderstudie Österreich) angemessen, deutschsprachige Titel dominieren. Selbst scheinbar randständige Quellen, die wichtige Informationen enthalten, sind von Sickinger akribisch verarbeitet worden.

Die große Stärke der Darstellung liegt im enzyklopädischen Charakter. Wer wissen will, welche Formen der Politikfinanzierung es gibt oder wie einzelne Möglichkeiten in Österreich praktiziert werden, sollte dieses Nachschlagewerk zur Hand haben. Wie vom Autor versprochen liefert das Buch für Journalisten, politische Praktiker und Wissenschaftler eine umfassende empirische Bestandsaufnahme der Politikfinanzierung in Österreich im Kontext internationaler Forschungsbefunde. Die Präsentation verbindet problemorientierte, hochverdichtete Beschreibung mit Quantifizierung durch langfristige Zeitreihen und systematischer Diskussion von Reformperspektiven. Bewertungsmaßstäbe für die Rolle des Gel- 
des als Ressource im Parteienwettbewerb sind seine traditionellen Risiken: Korruptionsgefahr, verzerrte Wettbewerbschancen der Parteien und Umsetzung sozialer Ungleichheit in politische Entscheidungen. Beim Kampf gegen diese Risiken demokratischer Politik spielt Transparenz der Politikfinanzierung eine zentrale Rolle.

Gerade ein an Österreich wenig interessierter Leser findet in den abschließenden (durchaus landesbezogen formulierten) Kapiteln eine Fülle von Ansatzmöglichkeiten für die Betrachtung des eigenen Landes oder vergleichende Analysen. Zunächst werden Maßstäbe vorgeführt, deren Einsatz helfen kann, die tatsächlichen Kosten der Parteiendemokratie differenziert zu beurteilen. Dekonzentration des Parteiensystems, Bedeutungsverlust der Parteiorganisationen, Bedeutungszuwachs der Medien und veränderte Medienlandschaft sind keine Besonderheiten der österreichischen Politik, wirken aber auf die Politikfinanzierung ein. Daraus werden oft Sachzwänge für die Kostenentwicklung abgeleitet. Sickinger vermeidet solche Pauschalthesen und zeigt ein Primat der Politik (und damit Handlungsmöglichkeiten) für die tatsächliche Ausgestaltung der Politikfinanzierung auf. Ausführlich und differenziert setzt er sich mit den vielfach behaupteten Auswirkungen der öffentlichen Parteienfinanzierung auseinander. Als Verfestigung des Parteiensystems konstatiert er vorrangig ein Festhalten am parteiorganisatorischen Status quo. Die finanzielle Autonomie der Parteileitungen (von den Parteimitgliedern) ist aber auch eine verstärkte Orientierung der Parteieliten an der Wählerschaft. Wichtige Änderungen des Parteialltags (nämlich Professionalisierung und Bürokratisierung) werden allerdings vom Konstrukt Etatisierung nicht gemessen. Gegen die Korruptionsgefahr ist öffentliche Parteienfinanzierung nur hilfreich im Zusammenwirken mit öffentlicher Kontrolle und einer medienvermittelten Abschreckung durch politische Skandale. Kurzum: Als Problemlösung ist die staatliche Politikfinanzierung nur ein möglicher Faktor, der vor allem verstärkend wirken kann.

Karl-Heinz Naßmacher

\section{Gewichtiger Jubiläumsband zu Extremismus und Demokratie}

Backes, Uwe und Eckhard Jesse (Hrsg.): Jahrbuch Extremismus \& Demokratie 2008, 20. Jahrgang, Nomos Verlagsgesellschaft, Baden-Baden 2009, 486 Seiten, € 49,-.

Der 20. Band des Jahrbuches „Extremismus und Demokratie“ stellt einen wahren Jubiläumsband dar: Das „60/20“-Jubiläum von provisorischer Reorganisation des Staates im Westen und friedlicher Revolution im Osten setzt den Rahmen. Außerdem wird Bezug genommen auf die Studentenunruhen vor 40 Jahren, auf die 30. Wiederkehr des „deutschen Herbstes“ und die 25 Jahre zurückliegende Gründung der „Marxistisch-Leninistischen Partei Deutschlands“. Die 486 Seiten teilen sich in 97 Seiten reflektierende „Analysen“, 137 Seiten faktenreiche „Daten, Dokumente, Dossiers“ und einen umfangreichen Literaturteil (212 Seiten), ergänzt um Einführung und Personenregister. Wiederum sind sowohl Nachwuchskräfte als auch namhafte Wissenschaftler als Autoren gewonnen worden. Erfreulicherweise verwendet das Jahrbuch weiterhin die herkömmliche Orthographie.

Zwei überblicksartig angelegte Artikel der Herausgeber dokumentieren 60 Jahre Bundesrepublik Deutschland als Periode extremistischer Herausforderungen einerseits, 